\title{
2014 Gauss Prize Awarded
}

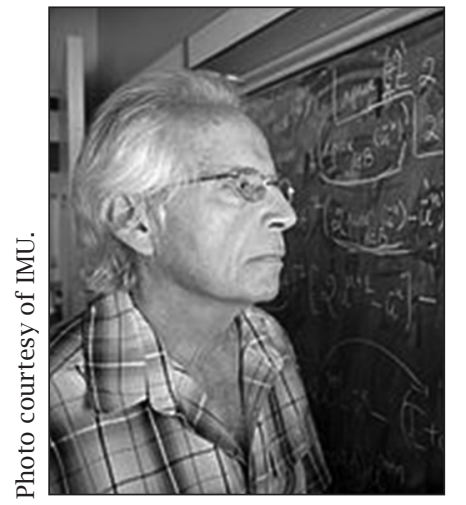

Stanley Osher
On August 13, 2014, the 2014 Carl Friedrich Gauss Prize for Applications of Mathematics was awarded at the opening ceremonies of the International Congress of Mathematicians (ICM) in Seoul, Korea. The prizewinner was STANLEY OSHER of the University of California Los Angeles "for his influential contributions to several fields in applied mathematics and for his far-ranging inventions that have changed our conception of physical, perceptual, and mathematical concepts, giving us new tools to apprehend the world."

The Carl Friedrich Gauss Prize for Applications of Mathematics was instituted in 2006 to recognize mathematical results that have opened new areas of practical applications. It is granted jointly by the International Mathematical Union and the German Mathematical Society and is awarded every four years at the ICM.

Stanley Osher is a one-man bridge between advanced mathematics and practical, real-world problems. Time and time again he has engaged deeply with the world of engineers and applied scientists and then developed mathematical techniques to solve their problems with unprecedented power, speed, and elegance. And because he so finely tunes his solutions to their needs, his techniques have been widely adopted and have had an extraordinarily broad impact, helping to catch criminals, create animated movies, improve MRI scans, design computer chips, and much more.

One of his major contributions was developing the method of level sets with James Sethian. The basic problem is to mathematically describe a changing shape. Imagine, for example, that a drop of oil is floating in water, and you want to write an equation that will predict how its shape will change with currents in the water. A traditional way to do this is to picture a set of buoys along the edge of the drop, connected by stretchy ropes.

DOI: http://dx.doi.org/10.1090/noti1179
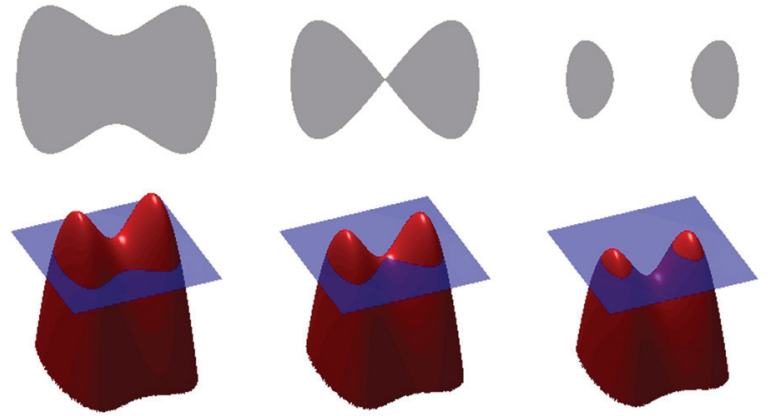

Horizontal slices of the three-dimensional red object on the bottom show the shape of an oil drop as it changes over time. This representation can gracefully handle behavior like the drop cleaving in two. Credit: Wikimedia.

Moment by moment, you can then describe how the buoys move, giving you a good approximation of the overall shape.

That method doesn't work so well, though, if your oil drop splits in half, as they sometimes do. It's not so obvious how to describe that with the buoys, since you now also need to cut the ropes between them and retie them. Or, suppose that two different oil drops merge-then some of your buoys disappear completely or fall inside the other oil drop or something. It gets messy.

Osher and Sethian suggested an entirely new approach. They imagine that the outline of the oil drop is a clean horizontal slice of some threedimensional object. The rest of the shape of the object doesn't much matter, as long as the slice is the right shape. They then apply the physical laws affecting the drop of oil (for the currents in the water, for example) to the entire three-dimensional

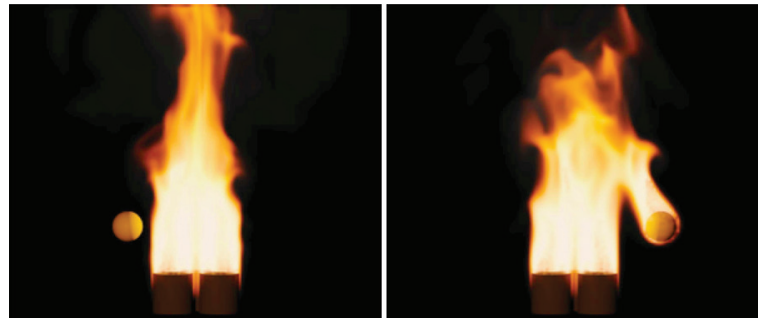

This is not a photograph. This is a computer simulation of a ball flying through a flame and catching fire. It was created using Osher's level set methods. Credit: Ron Fedkiw, Duc Nguyen, and Doug Enright. 

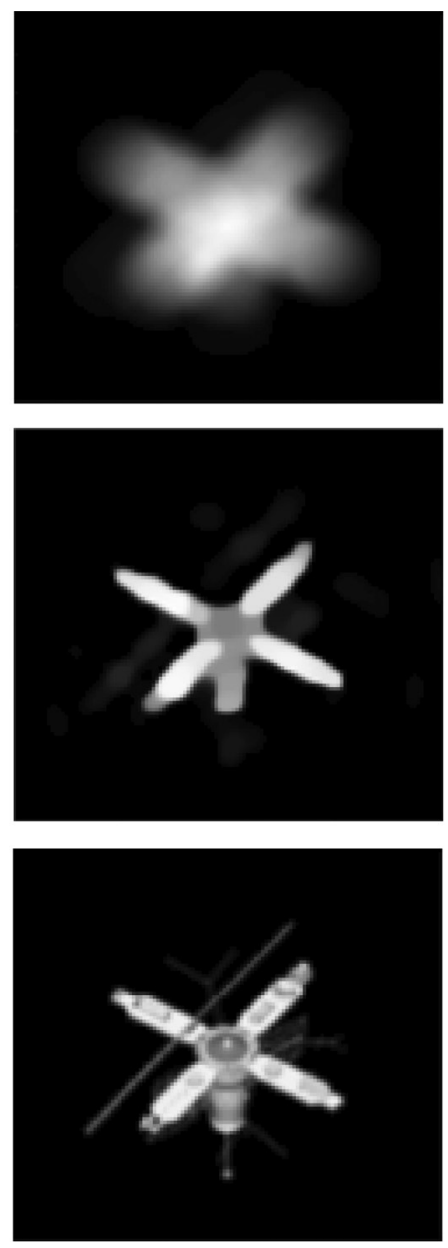

The top image is a blurry picture of TK. Osher's methods sharpen it into the middle image. The bottom image is a picture of the same thing that is in focus.

object. To find the shape of the oil drop at another moment, they simply take the same horizontal slice of the object later. If the oil drop splits, the three-dimensional object has developed two separate humps. And if two drops come together, two "legs" of the object have converged.

Although turning the problem of predicting the evolution of a two-dimensional object into predicting that of a three-dimensional one seems like it would only make things more complicated, it solves all the bookkeeping problems with the buoy-andropes method and gives a simple, powerful, clean representation that can handle any odd thing the oil drop might do.

This relatively simple idea turns out to be extremely powerful. For example, the level set method is now being used by every single major film animation company to animate fluids, including Pixar, Disney, ILM, Dreamworks, and more. It allows animators to apply the true laws of physics to the fluid, creating far more realistic images. The giant whirlpool maelstrom in Pirates of the Caribbean 3 and the dragon's flaming breath in Harry Potter and the Goblet of Fire, for example, were created using level sets. One of Osher's students, Ron Fedkiw of Stanford University, won an Academy Award for his work on film animation using these methods.

More practically, level set methods are useful for predicting weather, designing computer chips, identifying the source of an earthquake, modeling the growth of tumors, analyzing medical scans, and much, much more.

Another area that Osher has revolutionized is modeling the way that supersonic jets slice through the air. Air flows smoothly around planes flying at ordinary speeds, but when jets approach the speed of sound, the air can't get out of the way fast enough. As a result, the density, pressure, temperature, and velocity of the air change essentially instantaneously. In mathematical terms, this instantaneous change is called a "discontinuity," and it's a really big problem, because traditional methods all depend on incremental changes. Osher, together with Amiram Harten, Bjorn Engquist, and Chi-Wang Shu, developed new mathematical tech- niques that can handle these discontinuities. This enables computer modeling of the design of new supersonic jets.

Osher then applied similar ideas to an entirely different problem: sharpening blurry images. The line in a photograph between the dark leg of a table and a light background is another example of a discontinuity. Together with Leonid Rudin, Osher applied the mathematical techniques he had developed for modeling supersonic jets to images and was able to pull out and sharpen these discontinuities.

Their techniques also came from the recognition that much of the fuzz in a blurry image isn't random: it comes from physical processes like the shake of the hand holding the camera. Identifying these processes and then reversing them reduces the blur. That's easier said than done, though, because information is lost when the image is blurred. Osher's methods can recover some of that by combining information from multiple images. And he developed very efficient algorithms to carry out the transformation.

Never content with merely developing theoretical methods, Osher (together with Rudin) created a company, Cognitech, to commercialize them. The most famous success of the company came during a trial after the 1992 Los Angeles riots. Rioters attacked a truck that happened to be driving through the area, throwing rocks at it, dragging the driver out of the cab, beating him to unconsciousness, and breaking his skull in ninety-one places. The entire attack was filmed by a TV helicopter (one of the attackers even danced over the victim's unconscious body and flashed gang symbols to the helicopter). The footage was blurry, though, so prosecutors turned to Cognitech for help identifying the attackers. Investigators focused on a speck on the arm of one of the men-less than $1 / 6,000$ th the size of the total photograph-and the algorithms revealed it to be a rose-shaped tattoo. The man was later identified as Damian Monroe Williams, and he was convicted of the attack. Cognitech continues to be used by police departments across the country.

Another of Osher's clever algorithms allows people to get better MRI scans faster. Building on the compressive sensing ideas of David Donoho, Emmanuel Candès, and Terence Tao, he generalized his image-processing methods to any situation in which you want to present information using as little data as possible. Jpeg, for example, is an algorithm that stores images using fewer bytes while losing only a small amount of detail. He developed algorithms to do the same thing in reverse, so that the scan gathers its data in compressed format, requiring less of it to get a clear view. Another example where these techniques apply is the "Netflix problem," predicting what movies you will like from your favorites. The movies you've 
seen and liked are the data represented in compressed form, and the problem is to uncompress that to discover all the movies you will like.

As diverse as Osher's impacts on the world have been, they've all come from clever, efficient algorithms drawing on deep mathematics. "I write the algorithms that make the computer sing," Osher told the Los Angeles Times. "I'm the Barry Manilow of mathematics."

Stanley Osher was born in Brooklyn, New York, in 1942 and received his B.S. from Brooklyn College in 1962 and his Ph.D. from New York University in 1966. He worked at Brookhaven National Laboratories (1966-68) and as assistant professor at the University of California Berkeley from 1968 to 1970. He has been associate professor (1970-75) and professor (1975-77) at the State University of New York at Stony Brook. He has been a professor in the Department of Mathematics at UCLA since 1977 and is also director of special projects at the Institute for Pure and Applied Mathematics (IPAM). He has been a Fulbright Fellow (1971) and an Alfred P. Sloan Fellow (1972-74). He has received the Japan Society of Mechanical Engineers 2002 Computational Mechanics Award, the 2003 ICIAM Pioneer Prize, and the 2007 United States Association for Computational Mechanics (USACM) Computational and Applied Sciences Award, as well as the Kleinman Prize (2005) and the John von Neumann Lectureship (2013), both from the Society for Industrial and Applied Mathematics (SIAM). He has been an invited speaker (1994) and a plenary speaker (2010) at the International Congress of Mathematicians (ICM). He is a member of the National Academy of Sciences and the American Academy of Arts and Sciences, as well as a fellow of SIAM and of the AMS.

Awarded every four years at the International Congress of Mathematicians, the Gauss Prize carries a cash award of 10,000 euros (about US\$14,000). Previous recipients are Kiyoshi Itô(2006) and Yves Meyer (2010). On the selection committee for the 2014 prize were Alfio Quarteroni (chair), Weinan E, Barbara Keyfitz, Andrés Weintraub, and Aad van der Vaart.

- From an IMU news release
FROM THE CENTER FOR THE STUDY OF LANGUAGE AND INFORMATION

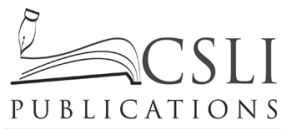

\section{NEW STUDIES IN WEAK ARITHMETICS}

\section{Edited by PATRICK CÉGIELSKI, CHARALAMPOS CORNAROS, and COSTAS DIMITRACOPOULOS}

This volume is dedicated to late Australian mathematician Alan Robert Woods whose seminal thesis is published here for the first time. The collection also contains other original papers on topics addressed in Woods' thesis and life's work.

Paper $\$ 35.00$

Distributed by the University of Chicago Press www.press.uchicago.edu
䍐

\section{UNIVERSITYATALBANY}

ate University of New York

\section{Dept. of Mathematics and Statistics Tenure-track Positions}

The Department of Mathematics and Statistics at the University at Albany, State University of New York, invites applications for two tenure-track assistant professor positions, one in algebra and one in analysis, both broadly construed, to start in fall 2015 .

We are looking for candidates who will significantly contribute to the department's research, closely collaborate with existing members of the department, and enhance our undergraduate and graduate programs.

Candidates should possess excellent research credentials as demonstrated by their $\mathrm{PhD}$ dissertation, publications, external funding, and as supported by letters of recommendation from experts in the field. Also of great importance are teaching credentials demonstrated by student evaluations and/or teaching awards and supported by letters of recommendation.

Candidates are required to have a $\mathrm{PhD}$ or an equivalent doctoral degree in Mathematics from a university accredited by the U.S. Department of Education or an internationally recognized accrediting organization. Postdoctoral experience and a successful record of external funding are highly desirable. All candidates must address in their applications their ability to work with a culturally diverse population and should provide statements on teaching and research.

Candidates are required to apply using the University employment portal. Please use: http://albany.interviewexchange.com/jobofferdetails.jsp? JOBID=52939 for the algebra position, and use http://albany.interviewexchange.com/jobofferdetails.jsp?JOBID=52938 for the analysis position.

Please also have at least four letters of recommendation sent to the Chair, Department of Mathematics and Statistics, University at Albany, Albany, NY 12222. At least two letters should address the candidate's research and at least one should address the candidate's teaching. These letters can also be emailed to kzhu@albany.edu. The deadline for applications is January 15, 2015.

The University at Albany is an EO/AA/IRCA/ADA Employer. 